\title{
Novel variants of the qnrB gene, qnrB31 and qnrB32, in Klebsiella pneumoniae
}

\begin{abstract}
Correspondence
Dongguo Wang

wdgtzs@163.com

Yongxiao Oi

qyxzlt@163.com
\end{abstract}

Received 15 May 2011

Accepted 1 August 2011

\author{
Dongguo Wang, ${ }^{1}$ Haibao Wang, ${ }^{1}$ Yongxiao ${ }^{2}{ }^{2}{ }^{2}$ Yong Liang, ${ }^{2}$ Jing Zhang ${ }^{1}$ \\ and Lianhua $\mathrm{Yu}^{1}$
}

\author{
${ }^{1}$ Department of Clinical Laboratory Medicine, Medical College of Taizhou University Affiliated \\ Taizhou Municipal Hospital, Taizhou, ZheJiang Province, PR China \\ ${ }^{2}$ Department of Laboratory Medicine, Medical College of Taizhou University, Taizhou, ZheJiang \\ Province, PR China
}

\begin{abstract}
Quinolone resistance in the family Enterobacteriaceae is mostly attributed to the accumulation of mutations in the bacterial enzymes targeted by fluoroquinolones: DNA gyrase and DNA topoisomerase IV. Here we isolated the Klebsiella pneumoniae strains KP3606 and KP4707 from different specimens from 2008 to 2010 in Taizhou Municipal Hospital of China, and discovered a new subtype qnrB31, for which the GenBank accession number is HQ418999, and another new subtype qnrB32, for which the GenBank accession number is HQ704413. Susceptibility testing showed that KP3606 had a reduced susceptibility ( $\mathrm{MIC} \geqslant 0.5 \mu \mathrm{g} \mathrm{ml}^{-1}$ ) to quinolones, while KP4707 was resistant to quinolones. Of all qnrB alleles, the novel variants the qnrB32 gene and qnrB31 gene have the highest amino acid identity. The results suggested that of all the various genes involved in resistance to quinolones, the $q n r B$ gene is the most likely to be mutated, and plasmids might play a role in the dissemination and evolution of $q n r B$ genes.
\end{abstract}

\section{INTRODUCTION}

Quinolones are synthetic broad-spectrum antibiotics used widely to treat a variety of infectious diseases. Bacterial resistance to quinolones emerged rapidly after their widespread use. In Gram-negative bacteria, quinolone resistance was for a long time considered to be entirely mediated by mutations in chromosomal genes encoding quinolone targets (that is, DNA gyrase and topoisomerase IV) and/or in regulatory genes of outer-membrane proteins or efflux pumps (Jacoby, 2005).

The first plasmid-encoded quinolone resistance gene ( $q n r$ ) was discovered in a Klebsiella pneumoniae isolate from Birmingham, Alabama, USA, collected in 1994. It occurred in a multi-resistance plasmid, pMG252, in an integron-like structure near orf513. Qnr, the gene product, is a member of the pentapeptide repeat protein (PRP) family, and has been shown to block the action of ciprofloxacin on purified DNA gyrase and topoisomerase IV (Tran \& Jacoby, 2002; Tran et al., 2005). Qnr plasmids have been found in clinical isolates of Citrobacter freundii, Enterobacter spp., Escherichia coli, K. pneumoniae, Providencia stuartii and Salmonella spp., from the United States, Europe, and the Near and Far East

Abbreviations: PMQR, plasmid-mediated quinolone resistance; PRP, pentapeptide repeat protein.

The GenBank/EMBL/DDBJ accession numbers for the genomic DNA sequences of qnrB31 and qnrB32 are HQ418999 and HQ704413, respectively.
(Cheung et al., 2005; Nordmann \& Poirel, 2005). Another $q n r$ gene, qnrS, has also recently been found in a plasmid from a strain of Shigella flexneri isolated in Japan (Kim et al., 2010). Since then, qnr alleles have been discovered worldwide in clinical strains of Gram-negative bacilli. The Qnr proteins confer quinolone resistance, and belong to the PRP family (Guo et al., 2010).

Plasmid-encoded quinolone resistance has been described along with three mechanisms: (i) a quinolone-protective mechanism encoded by the qnr genes; (ii) a modifying enzyme, aac(60)-Ib-cr (Robicsek et al., 2006a); and (iii) an efflux pump encoded by the qepA gene (Périchon et al., 2007).

Recently, plasmid-mediated quinolone resistances (PMQRs) have been reported worldwide in unrelated enterobacterial species associated with mobile elements (Robicsek et al., 2006b). Quinolones and $\beta$-lactams are among the most commonly used antimicrobials in human therapeutics. PMQR determinants confer low-level resistance, but their presence could potentially facilitate the evolution of the bacterial host toward higher levels of resistance by mutational alterations in type II topoisomerases. PMQR determinants are often combined with extended-spectrum $\beta$-lactamases suggesting the potential for selection of PMQR by the use of $\beta$-lactams and of $\beta$-lactam resistance by the use of quinolones (Strahilevitz et al., 2009; Guillard et al., 2010). 
Here, we researched the distribution and characteristics of K. pneumoniae $\mathrm{PMQR}$ qnrB genes, and characterized $q n r B$ genes, qnrB31 and qnrB32.

\section{METHODS}

Strains. A total of 72 isolates of K. pneumoniae were isolated from different specimens from 2008 to 2010 in Taizhou Municipal Hospital, China. Two isolates, KP3606 and KP4047, were found to exhibit resistance to quinolone and cephalosporin. The isolates were further used for susceptibility testing.

Conjugation and susceptibility testing. Conjugation experiments were carried out in Luria-Bertani (LB) broth with E. coli J53 Azide ${ }^{\mathrm{R}}$ (a strain with resistance to sodium azide) as the recipient, a PCR positive strain was used as the donor strain (Wang et al., 2003). Cultures of donor and recipient cells in exponential phase $(0.5 \mathrm{ml}$ of each) were added to $4 \mathrm{ml}$ fresh $\mathrm{LB}$ broth and incubated overnight without shaking. Transconjugants were selected on trypticase soy agar plates supplemented with $300 \mathrm{mg}$ sodium azide $\mathrm{l}^{-1}$ and $0.03 \mathrm{mg}$ ciprofloxacin $1^{-1}$, and incubated for $18-24 \mathrm{~h}$ at $35^{\circ} \mathrm{C}$. The susceptibility was assessed by MIC values. To determine if quinolone resistance was co-transferred, the MICs for antibiotics for the donor, recipient and transconjugant strains were compared (Kim et al., 2010). The MICs were determined by broth dilution and interpreted according to Clinical and Laboratory Standards Institute (formerly the National Committee for Clinical Laboratory Standards) guidelines (NCCLS, 2003).

PCR amplification and sequencing. To carry out the genetic characterization of the $q n r B$ allele gene in $K$. pneumoniae and its transconjugants (KP3606-ECJ53 Azide ${ }^{\mathrm{R}}$ and KP4707-ECJ53Azide ${ }^{\mathrm{R}}$ transconjugants), qnrB31 and qnrB32 were amplified by PCR, using primers F $5^{\prime}$-ATGTTGTCATTACTGTATA- $3^{\prime}$ and $\mathrm{R} \quad 5^{\prime}$-CTAA CCAATCACAGCGATG-3', and sequencing was carried out using primers $\mathrm{F}$ 5'-ATG ACGCCATTACTGTATAAAAAA-3' and R 5'-CTAGCCAATAATCGCGATGCCA-3'. Transconjugants (KP3606ECJ53 Azide $^{\mathrm{R}}$ and KP4707-ECJ53Azide ${ }^{\mathrm{R}}$ transconjugants) were amplified and sequenced by PCR using primers $\mathrm{F} 5^{\prime}$-ATGACTCTGGCGTTAGTTGG-3' and $\mathrm{R}$ 5'-TTAACCCATGACAGCGATACCAA- $3^{\prime}$, then the sequencing results were assembled to obtain the full-length nucleotides of qnrB31 and qnrB32 in K. pneumoniae and its transconjugants. Three pairs of primers were designed internal to the qnrB ORF, which allowed easy detection of the mutant alleles.

Gene mapping. To study the plasmids carrying the qnrB31 and qnrB32 genes, plasmid DNA was extracted and separated by $0.6 \%$ agarose gel electrophoresis, and then different sized fragments were cut out of the gel and purified. PCR was conducted to amplify qnrB gene using this plasmid DNA as the template, where the initial position of the plasmid $q n r B$ gene was determined, and size of the plasmid DNA estimated (Wang et al., 2003). The presence of PMQR determinants and quinolone resistance-determining region mutations was investigated by PCR-based detection and sequencing (Cano et al., 2009; Eaves et al., 2004; Jeong et al., 2004).

\section{RESULTS}

\section{Susceptibility testing}

The conjugation experiments of qnrB31 gene were carried out nine times while qnrB32 experiments were carried out six times until successful. The susceptibility testing results showed that the MIC values for quinolones for the transconjugants (KP3606-ECJ53 Azide $^{\mathrm{R}}$ and KP4707ECJ53 Azide $^{\mathrm{R}}$ transconjugants) were higher than that of $E$. coli J53 Azide ${ }^{\mathrm{R}}$. Furthermore, the MIC values for quinolones for the transconjugant carrying qnrB32 (KP4707-ECJ53 Azide $^{\mathrm{R}}$ transconjugant) was higher than that for the transconjugant harbouring qnrB31 (KP3606-EC)53 Azide ${ }^{\mathrm{R}}$ transconjugant). Conjugation experiments were successful and indicated that quinolone resistance was caused by the plasmid-encoded gene. Characterization of the qnrB-harbouring plasmids is shown in Fig. 1.

The transconjugate isolates of $K$. pneumoniae/E. coli showed resistance to ofloxacin, levofloxacin, ciprofloxacin and nalidixic acid. K. pneumoniae KP3606 showed a reduced susceptibility $\left(\mathrm{MIC} \geqslant 0.5 \mu \mathrm{g} \mathrm{ml}^{-1}\right.$ ) to quinolones (nalidixic acid, ofloxacin, levofloxacin and ciprofloxacin) (Table 1), while K. pneumoniae KP4707 was resistant to quinolones (Table 1).

\section{qnrB gene alleles and gene mapping}

Sequence analysis identified two novel qnrB variants, qnrB31 (GenBank accession number HQ418999) and qnrB32 (GenBank accession number HQ704413), in K. pneumoniae. The new variant $q n r B 31$ allele was assigned according to the qnr numbering scheme shown on the Lahey website (http:// www.lahey.org/qnrStudies), and the new variant qnrB32 allele sequence was released to the public on 22 February 2011 by GenBank. Based on gene mapping, there were three different length plasmids in isolates of KP3606 and KP4707. The qnrB31 and qnrB32 genes were separately located in an about $23.1 \mathrm{~kb}$ length plasmid.

\section{Amino acid point mutation analysis}

The amino acid point mutations associated with quinolone resistance were detected in the two isolates (Table 1). Six

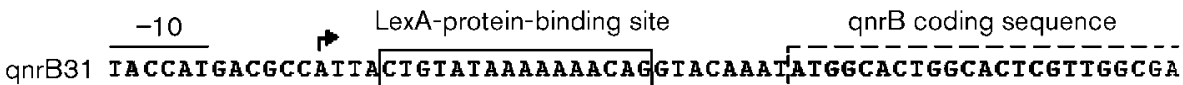

$$
\begin{aligned}
& \text { qniB32 TA CCATGACGCCATTACTGTATAAgAaAACAGTACAAATATGGCTCTGgCGCTCGTGgGCGA }
\end{aligned}
$$

Fig. 1. Characterization of the $q n r B$-harbouring plasmids. The -10 promoter element is indicated; the +1 start site is represented by an arrow. The start of the qnr $B$ coding sequence is indicated by dashed lines and the consensus sequence of the LexA-protein-binding site is boxed. The LexA-protein-binding site was the determining feature of fluoroquinolone resistance. If the LexA-protein-binding site was present then strains were sensitive to fluoroquinolone, and vice versa. 
Table 1. Characteristics of the two K. pneumoniae isolates and of the E. coli J53 Azide ${ }^{\mathrm{R}}$ clone harbouring the qnrB31 and qnrB32 genes

\begin{tabular}{|c|c|c|c|c|c|c|}
\hline \multirow[t]{2}{*}{ Strain } & \multirow{2}{*}{$\begin{array}{c}\text { Amino acid point } \\
\text { mutations }{ }^{\star}\end{array}$} & \multirow{2}{*}{$\begin{array}{c}\text { PMQR } \\
\text { determinant }\end{array}$} & \multicolumn{4}{|c|}{$\operatorname{MIC}\left(\mu \mathrm{g} \mathrm{ml}^{-1}\right) \dagger$} \\
\hline & & & NAL & OFL & LVN & CIP \\
\hline \multicolumn{7}{|c|}{ K. pneumoniae } \\
\hline KP3606 & $\begin{array}{l}\text { Arn } 27 \rightarrow \text { Leu } \\
\text { Ser79 } \rightarrow \text { Ala } \\
\text { Arg87 } \rightarrow \text { Ser } \\
\text { Ile142 } \rightarrow \text { Met } \\
\text { Gly188 } \rightarrow \text { Arg } \\
\text { Val212 } \rightarrow \text { Ile }\end{array}$ & $q n r 31$ & $>256$ & 16 & 8 & 4 \\
\hline KP4707 & $\begin{array}{l}\text { Ser79 } \rightarrow \text { Ala } \\
\text { Val129 } \rightarrow \text { Ala } \\
\text { Ile142 } \rightarrow \text { Met } \\
\text { Gly188 } \rightarrow \text { Arg } \\
\text { Val212 } \rightarrow \text { Ile }\end{array}$ & qnr32 & $>256$ & 32 & 16 & 8 \\
\hline \multicolumn{7}{|c|}{ Transconjugants } \\
\hline $\begin{array}{r}\text { KP3606- } \\
\text { transcor }\end{array}$ & & $q n r 31$ & 16 & 0.5 & 0.125 & 0.0625 \\
\hline $\begin{array}{r}\text { KP4707- } \\
\text { transcor }\end{array}$ & & $q n r 32$ & 32 & 2 & 0. 25 & 0.125 \\
\hline E. coli $\mathrm{J} 5$ & & - & 2 & 0.0039 & 0.00195 & 0.00195 \\
\hline
\end{tabular}

${ }^{*}$ Amino acid point mutations are compared with qnrB31 and qnrB32 (each other).

$\dagger$ NAL, Nalidixic acid; OFX, ofloxacin; LVX, levofloxacin; CIP, ciprofloxacin.

amino acid mutations were observed in the qnrB31 gene, they were Arn27 $\rightarrow$ Leu, Ser79 $\rightarrow$ Ala, Arg87 $\rightarrow$ Ser, Ile142 $\rightarrow$ Met, Gly188 $\rightarrow$ Arg and Val212 $\rightarrow$ Iie when compared with the amino acid sequence of $q n r B 1$. Five point mutations were observed in the qnrB32 gene (Ser79 $\rightarrow$ Ala, Val129 $\rightarrow$ Ala, Iie142 $\rightarrow$ Met, Gly188 $\rightarrow$ Arg and Val212 $\rightarrow$ Iie) compared with the amino acid sequence of qnrB1.

The qnrB31 gene had $91.6 \%$ nucleotide identity with qnrB1. The qnrB32 gene had $90.3 \%$ nucleotide identity with qnrB1. The qnrB31 gene had $97.1 \%$ nucleotide identity with qnrB32. The qnrB allele base sequence indicated that qnrB32 (HQ704413) and qnrB31 (HQ418999) were homogeneous, with 100 support value.

\section{DISCUSSION}

This study was carried out to determine the prevalence of $q n r$ genes among 72 consecutive non- repetitive Enterobacteriaceae of patient origin and to characterize the positive isolates. The presence of PMQR determinants and quinolone resistance-determining region mutations was investigated, and the $q n r A$, qnrS, qnrC, qnrD, aac( $\left.6^{\prime}\right)-I b-c r$ and $q e p A$ genes were not found. Two isolates of K. pneumoniae, KP3606 and KP4707, were found to carry $q n r$-like genes that were not found in E. coli. Sequence analysis identified two novel $q n r B$ variants, named qnrB31 (HQ418999) and qnrB32 (HQ704413).
Plasmid-encoded genes, such as qnr, may facilitate the spread and increase of the prevalence of quinoloneresistant strains. To date, qnr genes have been widely evaluated in southern and eastern Asia, North and South America, and Europe (Martínez-Martínez et al., 1998; Tran \& Jacoby, 2002), and the $q n r$ gene has been shown to block the action of ciprofloxacin on purified DNA gyrase and topoisomerase IV (Tran \& Jacoby, 2002; Cattoir et al., 2008; Tran et al., 2005). qnrB provides low-level resistance to quinolones and belongs to the PRP family, one member of which has recently been shown to have a DNA-like structure that would allow it to mimic DNA as a substrate for DNA gyrase (Jiang et al., 2008; Hegde et al., 2005). For a protein in which overall structure is important rather than catalytic activity, considerable amino acid variability may be permissible.

An integrated $q n r B$ gene sequence included three parts as follows: a promoter sequence ( -35 to -10 region), the consensus sequence of the LexA-protein-binding site and the $q n r B$ coding sequence. If any antibiotic can open the consensus sequence of the LexA-protein-binding site, promoter sequence and $q n r B$ coding sequence this will result in quinolone resistance. Therefore, of these three parts, the presence of the consensus sequence of the LexAprotein-binding site was the key to quinolone resistant ( $\mathrm{Da}$ Re et al., 2009; Wang et al., 2009). The LexA-proteinbinging site was determining fluoroquinolone resistance. If the LexA-protein-binding site was present then those 
strains were sensitive to fluoroquinolone, and vice versa. In our analysis, the strains were quinolone resistant, which might indicate the consensus sequence of the LexAprotein-binding site was subject to autoproteolytic cleavage leading to quinolone resistance.

On induction of the SOS response, by ciprofloxacin for example, ssDNA is produced and the co-protease activity of the RecA protein is activated by binding to ssDNA. The interaction between LexA and the nucleoprotein filament RecA/ssDNA results in autoproteolytic cleavage of LexA and subsequently in $q \eta r B$ derepression. Induced expression of $q n r B$ leads to an increase in the ciprofloxacin MIC (Da Re et al., 2009).

Mutation in the gene sites induced bacterial quinolone resistance. Abuse of quinolone antibiotics could transmit to the next generation and lead to large numbers of quinolone-resistant bacteria. Therefore, clinical quinolone antibiotics could not be applied to infection. However, these phenomena might be temporary. If the use of quinolone antibiotics was reduced, the mutated genes could self-repair after several generations, then the rate of quinolone-resistant bacteria would be reduced.

In summary, the quinolone resistance genes qnr31 and qnr32 led to multi-drug resistance in K. pneumoniae KP4707 and KP3606. The abuse of broad-spectrum antibiotics would induce the emergence of resistant genes easily, resulting in multi-drug resistant bacteria, and attention should be paid to this.

\section{ACKNOWLEDGEMENTS}

The study was supported by a grant from the Medical College of Taizhou University Affiliated Taizhou Municipal Hospital and a grant from the Taizhou Science and Technology Bureau, Zhejiang, China (no. $081 \mathrm{KY} 30$ ).

\section{REFERENCES}

Cano, M. E., Rodríguez-Martínez, J. M., Agüero, J., Pascual, A., Calvo, J., García-Lobo, J. M., Velasco, C., Francia, M. V. \& Martínez-Martínez, L. (2009). Detection of plasmid-mediated quinolone resistance genes in clinical isolates of Enterobacter spp. in Spain. J Clin Microbiol 47, 2033-2039.

Cattoir, V., Nordmann, P., Silva-Sanchez, J., Espinal, P. \& Poirel, L. (2008). ISEcp 1-mediated transposition of qnrB-like gene in Escherichia coli. Antimicrob Agents Chemother 52, 2929-2932.

Cheung, T. K., Chu, Y. W., Chu, M. Y., Ma, C. H., Yung, R. W. \& Kam, K. M. (2005). Plasmid-mediated resistance to ciprofloxacin and cefotaxime in clinical isolates of Salmonella enterica serotype enteritidis in Hong Kong. J Antimicrob Chemother 56, 586-589.

Da Re, S., Garnier, F., Guérin, E., Campoy, S., Denis, F. \& Ploy, M. C. (2009). The SOS response promotes $q n r B$ quinolone-resistance determinant expression. EMBO Rep 10, 929-933.

Eaves, D. J., Randall, L., Gray, D. T., Buckley, A., Woodward, M. J., White, A. P. \& Piddock, L. J. (2004). Prevalence of mutations within the quinolone resistance-determining region of $g y r A, \operatorname{gyr} B$, parC, and parE and association with antibiotic resistance in quinolone-resistant Salmonella enterica. Antimicrob Agents Chemother 48, 4012-4015.
Guillard, T., Duval, V., Moret, H., Brasme, L., Vernet-Garnier, V. \& De Champs, C. (2010). Rapid detection of $a a c\left(6^{\prime}\right)-I b-c r$ quinolone resistance gene by pyrosequencing. J Clin Microbiol 48, 286-289.

Guo, Q., Weng, J., Xu, X., Wang, M., Wang, X., Ye, X., Wang, W. \& Wang, M. (2010). A mutational analysis and molecular dynamics simulation of quinolone resistance proteins QnrAl and QnrC from Proteus mirabilis. BMC Struct Biol 10, 33.

Hegde, S. S., Vetting, M. W., Roderick, S. L., Mitchenall, L. A., Maxwell, A., Takiff, H. E. \& Blanchard, J. S. (2005). A fluoroquinolone resistance protein from Mycobacterium tuberculosis that mimics DNA. Science 308, 1480-1483.

Jacoby, G. A. (2005). Mechanisms of resistance to quinolones. Clin Infect Dis 41 (Suppl. 2), S120-S126.

Jeong, S. H., Bae, I. K., Kwon, S. B., Lee, J. H., Jung, H. I., Song, J. S., Jeong, B. C., Kim, S. J. \& Lee, S. H. (2004). Investigation of extendedspectrum $\beta$-lactamases produced by clinical isolates of Klebsiella pneumoniae and Escherichia coli in Korea. Lett Appl Microbiol 39, 4147.

Jiang, Y., Zhou, Z., Qian, Y., Wei, Z., Yu, Y., Hu, S. \& Li, L. (2008). Plasmid-mediated quinolone resistance determinants $q n r$ and $a a c\left(6^{\prime}\right)-I b-c r$ in extended-spectrum $\beta$-lactamase-producing Escherichia coli and Klebsiella pneumoniae in China. J Antimicrob Chemother 61, 1003-1006.

Kim, H. B., Wang, M., Ahmed, S., Park, C. H., LaRocque, R. C., Faruque, A. S., Salam, M. A., Khan, W. A., Qadri, F. \& other authors (2010). Transferable quinolone resistance in Vibrio cholerae. Antimicrob Agents Chemother 54, 799-803.

Martínez-Martínez, L., Pascual, A. \& Jacoby, G. A. (1998). Quinolone resistance from a transferable plasmid. Lancet 351, 797-799.

NCCLS (2003). Methods for Dilution Antimicrobial Susceptibility Tests for Bacteria that Grow Aerobically, 6th edn, approved standard M7A6. Wayne, PA: National Committee for Clinical Laboratory Standards.

Nordmann, P. \& Poirel, L. (2005). Emergence of plasmid-mediated resistance to quinolones in Enterobacteriaceae. $J$ Antimicrob Chemother 56, 463-469.

Périchon, B., Courvalin, P. \& Galimand, M. (2007). Transferable resistance to aminoglycosides by methylation of G1405 in 16S rRNA and to hydrophilic fluoroquinolones by QepA-mediated efflux in Escherichia coli. Antimicrob Agents Chemother 51, 2464-2469.

Robicsek, A., Strahilevitz, J., Jacoby, G. A., Macielag, M., Abbanat, D., Park, C. H., Bush, K. \& Hooper, D. C. (2006a). Fluoroquinolonemodifying enzyme: a new adaptation of a common aminoglycoside acetyltransferase. Nat Med 12, 83-88.

Robicsek, A., Jacoby, G. A. \& Hooper, D. C. (2006b). The worldwide emergence of plasmid-mediated quinolone resistance. Lancet Infect Dis 6, 629-640.

Strahilevitz, J., Jacoby, G. A., Hooper, D. C. \& Robicsek, A. (2009). Plasmid-mediated quinolone resistance: a multifaceted threat. Clin Microbiol Rev 22, 664-689.

Tran, J. H. \& Jacoby, G. A. (2002). Mechanism of plasmid-mediated quinolone resistance. Proc Natl Acad Sci U S A 99, 5638-5642.

Tran, J. H., Jacoby, G. A. \& Hooper, D. C. (2005). Interaction of the plasmid-encoded quinolone resistance protein QnrA with Escherichia coli topoisomerase IV. Antimicrob Agents Chemother 49, 3050-3052.

Wang, M., Tran, J. H., Jacoby, G. A., Zhang, Y., Wang, F. \& Hooper, D. C. (2003). Plasmid-mediated quinolone resistance in clinical isolates of Escherichia coli from Shanghai, China. Antimicrob Agents Chemother 47, 2242-2248.

Wang, M., Jacoby, G. A., Mills, D. M. \& Hooper, D. C. (2009). SOS regulation of qnrB expression. Antimicrob Agents Chemother 53, 821-823. 